Article

\title{
New Existence of Fixed Point Results in Generalized Pseudodistance Functions with Its Application to Differential Equations
}

\author{
Sujitra Sanhan ${ }^{\circledR}$, Winate Sanhan and Chirasak Mongkolkeha * \\ Department of Mathematics, Statistics and Computer Sciences, Faculty of Liberal Arts and Science, \\ Kasetsart University, Kamphaeng-Saen Campus, Nakhonpathom 73140, Thailand; sujitrah@hotmail.com (S.S.); \\ faaswns@ku.ac.th (W.S.) \\ * Correspondence: chirasak.m@ku.th
}

Received: 8 November 2018; Accepted: 5 December 2018; Published: 12 December 2018

check for updates

\begin{abstract}
The purpose of this article is to prove some existences of fixed point theorems for generalized F-contraction mapping in metric spaces by using the concept of generalized pseudodistance. In addition, we give some examples to illustrate our main results. As the application, the existence of the solution of the second order differential equation is given.
\end{abstract}

Keywords: generalized pseudodistance; fixed point; $F$-contraction; differential equation

\section{Introduction}

In recent years, several generalizations of standard metric spaces have appeared in connection with generalizing a Banach contraction theorem. In 1996, Kada, Suzuki and Takahashi [1] defined the notions of a $w$-distance which is a generalized metric and also provided a generalized Caristi's fixed point theorem. Later, Wlodarczyk and Plebaniak [2] defined the notion of generalized pseudodistance which is generalized $w$-distance. In 2011, a fixed point results in uniform spaces for the Subrahmanyam type is proven [3]. In 2013, by using the concept of Meir-Keeler, Suzuki, Ćirić, Achymski, and Matkowski mappings, the fixed point results in uniform spaces were given [4]. Recently, Mongkolkeha and Kumam [5] used the notion of generalized weak contraction to prove the existence results of fixed point in $b$-metric spaces. On the other hand, in 2012, Wardowski [6] established a new contraction called $F$-contraction and acquired a fixed point result which generalized a Banach contraction in many ways. In 2014, Pili and Kumam [7] revealed a large class of functions by changing condition $(F 3)$ to $\left(F 3^{\prime}\right)$ in the condition of $F$ function. Afterwards, many authors used this mapping for studying the existences of fixed points and applied it to several problems [8-11]. Hence, by using the notion of $F$-contraction, we prove some new existence theorems of fixed points in generalized pseudodistances. Moreover, we also give some examples to support our main results. As an application, we establish the existence solution of a second order differential equation by using our fixed point results.

\section{Preliminaries}

In this section, we recall the notions, notations and basic knowledge for our consideration. First, we denote the sets of positive integers, positive real numbers and real numbers by $\mathbb{N}, \mathbb{R}^{+}$and $\mathbb{R}$, respectively.

Definition 1. Let $X$ be a nonempty set. A point $x \in X$ is called a fixed point of a mapping $T: X \rightarrow X$ if $x=T x$. We note that Fix $(T):=\{x \in X \mid x=T x\}$. 


\section{1. w-Distance and Generalized Pseudodistance}

Definition 2. Let $(X, d)$ be a metric space [1]. The mapping $p: X \times X \rightarrow[0, \infty)$ is said to be a w-distance on $X$ if it satisfies the following properties:

(a) $\quad p(x, z) \leq p(x, y)+p(y, z)$ for all $x, y, z \in X$;

(b) for any $x \in X, p(x, \cdot): X \rightarrow[0, \infty)$ is lower semi-continuous. That is,

$$
p(x, y) \leq \liminf _{n \rightarrow \infty} p\left(x, y_{n}\right)
$$

for any $x \in X$ and sequence $\left\{y_{n}\right\}$ in $X$ such that $y_{n} \rightarrow y \in X$.

(c) for each $\varepsilon>0$, there exists $\delta>0$ such that $p(z, x) \leq \delta$ and $p(z, y) \leq \delta$ imply $d(x, y) \leq \varepsilon$.

Obviously, every metric is a $w$-distance but not conversely (see [12]).

Definition 3. Let $(X, d)$ be a metric space and let $J: X \times X \rightarrow[0, \infty)$ be a mapping $[2,13]$. A mapping $J$ is called $a$ generalized pseudodistance on $X$ if it satisfies the following properties:

(J1) $J(x, y) \leq J(x, z)+J(z, y)$ for all $x, y, z \in X$,

(J2) for any sequences $\left\{x_{m}\right\}$ and $\left\{y_{m}\right\}$ in $X$ such that

$$
\lim _{n \rightarrow \infty} \sup _{m>n} J\left(x_{n}, x_{m}\right)=0
$$

and

$$
\lim _{m \rightarrow \infty} J\left(x_{m}, y_{m}\right)=0,
$$

we have

$$
\lim _{m \rightarrow \infty} d\left(x_{m}, y_{m}\right)=0
$$

For the examples of generalized pseudodistance, see $[2,13]$.

Remark 1. Let $(X, d)$ be a metric space and J be a generalized pseudodistance on $X$ :

(A) there exists a generalized pseudodistance J on $X$ which is not a metric.

(B) Every metric $d$ is a generalized pseudodistance on $X$.

(B) Let $X_{J}^{0}=\{a \in X: J(a, a)=0\}$ and $X_{J}^{+}=\{a \in X: J(a, a)>0\}$; then, $X=X_{J}^{0} \cup X_{J}^{+}$.

(C) For any $a, b \in X$ with $a \neq b$, then $J(a, b)>0 \vee J(b, a)>0$ (see ([13]).

Definition 4. Let $(X, d)$ be a metric space equipped with a generalized pseudodistance J [14]. We say that $X$ is a J-complete if and only if for all a sequence $\left\{x_{n}\right\}$ in $X$ such that

$$
\lim _{n \rightarrow \infty} \sup _{m>n} J\left(x_{n}, x_{m}\right)=0,
$$

there exists an element $x$ in X such that

$$
\lim _{m \rightarrow \infty} J\left(x_{m}, x\right)=\lim _{m \rightarrow \infty} J\left(x, x_{m}\right)=0
$$

\section{Remark 2.}

(A) If we take $J=d$, then the J-completeness reduces to the completeness.

(B) If $J=p$, when $p$ is a $w$-distances, we call $X$ a w-complete. 


\subsection{F-Contraction Mapping}

Definition 5. A function $F: \mathbb{R}^{+} \rightarrow \mathbb{R}$ is called F-function if it corresponds to the properties [6]:

(F1) $s<t \Rightarrow F(s)<F(t)$ for all $s, t \in \mathbb{R}^{+}$, i.e., $F$ is strictly increasing.

(F2) $\lim _{n \rightarrow \infty} s_{n}=0$ iff $\lim _{n \rightarrow \infty} F\left(s_{n}\right)=-\infty$, for every sequence $\left\{s_{n}\right\}$ in $\mathbb{R}^{+}$.

(F3) there exists a number $k \in(0,1)$ such that $\lim _{s \rightarrow 0^{+}} s^{k} F(s)=0$.

We denote that $\mathcal{F}$ is the set of all functions F-functions.

The examples of functions $F \in \mathcal{F}$ (see [6] for more details).

Definition 6. Let $(X, d)$ be a metric space [6]. A self mapping $T$ on $X$ is said to be an F-contraction if, for all $x, y \in X$ such that $d(T x, T y)>0$, we have

$$
\tau+F(d(T x, T y)) \leq F(d(x, y))
$$

for some $\tau>0$ and $F \in \mathcal{F}$.

Remark 3. Consider $F: \mathbb{R}^{+} \rightarrow R$ defined by $F(\alpha)=\ln \alpha$. Then, $F$ satisfies $(F 1)-(F 3)(F(3)$ for any $k \in(0,1))$. If $T: X \rightarrow X$ be a mapping satisfying (4), then

$$
d(T x, T y) \leq e^{-\tau} d(x, y)
$$

for all $x, y \in X$ with $T x \neq T y$. It is obvious that for $x, y \in X$, such that $T x=T y$, the inequality (4) also holds. That is, $T$ is Banach contraction.

Remark 4. Consider $F: \mathbb{R}^{+} \rightarrow R$ defined by $F(\alpha)=\ln \left(\alpha^{2}+\alpha\right)$. Then, $F$ satisfies (F1)-(F3) (F(3) for any $k \in(0,1))$. If $T: X \rightarrow X$ is a mapping satisfying (4), then

$$
\frac{d(T x, T y)(d(T x, T y)+1)}{d(x, y)(d(x, y)+1)} \leq e^{-\tau}
$$

for all $x, y \in X$ with $T x \neq T y$.

Remark 5. Let $F \in \mathcal{F}$. Then,

(a) By (F1) and (4) every F-contraction, $T$ is a contractive mapping. That is,

$$
d(T x, T y)<d(x, y)
$$

for all $x, y \in X$ with $T x \neq T y$.

(b) Every F-contraction is a continuous mapping.

In 2013, Secelean [10] showed that the condition (F2) in F-function can be by the equivalent condition instead but a more simple as follows:

$\left(F 2^{\prime}\right) \quad \inf F=-\infty$

or, instead, by

$\left(F 2^{\prime \prime}\right)$ there exists a sequence $\left\{s_{n}\right\}$ in $\mathbb{R}^{+}$such that $\lim _{n \rightarrow \infty} F\left(s_{n}\right)=-\infty$.

In 2014, Piri and Kumam [7] changed the property $(F 3)$ by $\left(F 3^{\prime}\right)$ in the F-function as follows. $\left(F 3^{\prime}\right) F$ is continuous on $(0, \infty)$. Thus, Piri and Kumam [7] generalized the result of Wordowski by 
using the conditions $(F 1),\left(F 2^{\prime}\right)$ and $\left(F 3^{\prime}\right)$. Recently, Singk et al. [11] removed the condition $\left(F 2^{\prime}\right)$ and it is called relaxed $F$ - contraction as follows.

Definition 7. A function $F: \mathbb{R}^{+} \rightarrow \mathbb{R}$ is called relaxed $F$ - function if it satisfies:

(F1) $F$ is strictly increasing,

$\left(F 3^{\prime}\right) \quad F$ is continuous on $(0, \infty)$.

We denoted that $\triangle_{\mathcal{F}}$ is the set of all relaxed F-functions.

\section{Main Results}

In this section, we prove the existence of fixed point theorems for generalized $F$-contraction mapping. In addition, we provide some examples to support our main results.

Theorem 1. Let $(X, d)$ be a metric space equipped with a generalized pseudodistance $J$ and let $T: X \rightarrow X$ be a mapping. Assume that $X$ is $J$-complete and there exists $F \in \mathcal{F}$ and $\tau \in \mathbb{R}^{+}$such that

$$
\tau+F(J(T x, T y)) \leq F\left(m_{J}(x, y)\right)
$$

for all $x, y \in X$ with $J(T x, T y)>0$, where $m_{J}(x, y)=\min \{J(x, y), J(y, x)\}$. If $F$ is a continuous from the right, then $T$ has a unique fixed point. Furthermore, for any $x_{0} \in X$, define $x_{n}=T x_{n-1}$ for all $n \in \mathbb{N}$, the sequence $\left\{x_{n}\right\}$ converges to $u \in \operatorname{Fix}(T)$ and $\lim _{n \rightarrow \infty} J\left(x_{n}, u\right)=0=\lim _{n \rightarrow \infty} J\left(u, x_{n}\right)$.

Proof. Let $x_{0} \in X$. We define the sequence $x_{n}$ by $x_{n}=T x_{n-1}$ for all $n \in \mathbb{N}$. If $x_{n_{0}}=T x_{n_{0}}$, for some $n_{0} \in \mathbb{N}$, the proof is complete. Suppose that $x_{n} \neq T x_{n}$ for all $n \in \mathbb{N}$ then we have $d\left(T x_{n-1}, T x_{n}\right)>0$. Consequently, $J\left(T x_{n-1}, T x_{n}\right)>0$. By (F1) and Equation (6), we have

$$
\tau+F\left(J\left(x_{n}, x_{n+1}\right)\right)=\tau+F\left(J\left(T x_{n-1}, T x_{n}\right) \leq F\left(m_{J}\left(x_{n-1}, x_{n}\right)\right),\right.
$$

putting $J_{n}:=J\left(x_{n}, x_{n+1}\right)$ for all $n \in \mathbb{N} \cup\{0\}$.

case I Let $m_{J}\left(x_{n-1}, x_{n}\right)=J\left(x_{n-1}, x_{n}\right)$, by Equation (7),

$$
F\left(J_{n}\right) \leq F\left(m_{J}\left(x_{n-1}, x_{n}\right)\right)-\tau=F\left(J_{n-1}\right)-\tau .
$$

Furthermore, by Equation (8) and (F1), we can see that

$$
F\left(J_{n}\right) \leq F\left(J_{n-1}\right)-\tau \leq F\left(J_{n-2}\right)-2 \tau \leq \ldots \leq F\left(J_{0}\right)-n \tau
$$

and thus $\lim _{n \rightarrow \infty} F\left(J_{n}\right)=-\infty$. From $(F 2)$, we have $\lim _{n \rightarrow \infty} J_{n}=0$. Now, using $(F 3)$, there exists $k \in(0,1)$ such that

$$
\lim _{n \rightarrow \infty} J_{n}^{k} F\left(J_{n}\right)=0
$$

By Equation (9), for each $n \in \mathbb{N}$, we get

$$
J_{n}^{k} F\left(J_{n}\right)-J_{n}^{k} F\left(J_{0}\right) \leq J_{n}^{k}\left(F\left(J_{0}\right)-n \tau\right)-J_{n}^{k} F\left(J_{0}\right) \leq-n \tau J_{n}^{k} \leq 0
$$

and hence

$$
\lim _{n \rightarrow \infty} n J_{n}^{k}=0
$$

Therefore, we can choose $n_{0} \in \mathbb{N}$ such that

$$
n J_{n}^{k} \leq 1 \text { for all } n \geq n_{0}
$$


and hence

$$
J_{n} \leq \frac{1}{n^{1 / k}} \quad \text { for all } n \geq n_{0}
$$

Letting $m, n$ be positive integer such that $m>n \geq n_{0}$, then

$$
\begin{aligned}
J\left(x_{n}, x_{m}\right) & \leq J\left(x_{n}, x_{n+1}\right)+J\left(x_{n+1}, x_{n+2}\right)+\ldots+J\left(x_{m-1}, x_{m}\right) \\
& =J_{n}+J_{n+1}+\ldots+J_{m-1} \\
& \leq \sum_{n \geq n_{0}} \frac{1}{n^{1 / k}}
\end{aligned}
$$

which is convergent as $k \in(0,1)$ and thus

$$
\lim _{n \rightarrow \infty} \sup _{m>n} J\left(x_{n}, x_{m}\right)=0
$$

case II Let $m_{J}\left(x_{n-1}, x_{n}\right)=J\left(x_{n}, x_{n-1}\right)$, then $J\left(x_{n}, x_{n-1}\right) \leq J\left(x_{n-1}, x_{n}\right)$.

By Equation (7) and (F1),

$$
\begin{aligned}
\tau+F\left(J\left(x_{n}, x_{n+1}\right)\right) & \leq F\left(m_{j}\left(x_{n-1}, x_{n}\right)\right) \\
& =F\left(J\left(x_{n}, x_{n-1}\right)\right) \\
& \leq F\left(J\left(x_{n-1}, x_{n}\right)\right)
\end{aligned}
$$

That is,

$$
F\left(J_{n}\right) \leq F\left(J_{n-1}\right)-\tau .
$$

By the same argument as in case I, we also get that Equation (12) holds. From the $J$-completeness of $J$, the sequence $\left\{x_{n}\right\}$ converges to some element $x_{*} \in X$ and hence

$$
\lim _{n \rightarrow \infty} J\left(x_{n}, x_{*}\right)=0=\lim _{n \rightarrow \infty} J\left(x_{*}, x_{n}\right) .
$$

Next, we will prove that $x_{*} \in F i x(T)$. If $J\left(T x_{n}, T x_{*}\right)>0$, by (F1) and Equation (6), we have

$$
\begin{aligned}
F\left(J\left(x_{n+1}, T x_{*}\right)\right) & =F\left(J\left(T x_{n}, T x_{*}\right)\right. \\
& \leq F\left(m_{J}\left(x_{n}, x_{*}\right)\right)-\tau .
\end{aligned}
$$

If $m_{J}\left(x_{n}, x_{*}\right)=J\left(x_{n}, x_{*}\right)$, then by Equation (14) and (F1), J( $\left.x_{n+1}, T x_{*}\right) \leq J\left(x_{n}, x_{*}\right)$. Hence, by Equation (13), we have

$$
\lim _{n \rightarrow \infty} J\left(x_{n+1}, T x_{*}\right) \leq \lim _{n \rightarrow \infty} J\left(x_{n}, x_{*}\right)=0 .
$$

Then,

$$
\lim _{n \rightarrow \infty} J\left(x_{n+1}, T x_{*}\right)=0 .
$$

For any $n \in \mathbb{N}$, define $x_{m}=x_{n+1}$ and $y_{m}=T x_{*}$ for all $m \in \mathbb{N}$; then,

$$
\lim _{m \rightarrow \infty} J\left(x_{m}, y_{m}\right)=0 .
$$

By Equations (12), (15) and (J2), imply that $\lim _{m \rightarrow \infty} d\left(x_{m}, y_{m}\right)=0$. That is,

$$
d\left(x_{*}, T x_{*}\right)=\lim _{n \rightarrow \infty} d\left(x_{n+1}, T x_{*}\right)=\lim _{m \rightarrow \infty} d\left(x_{m}, y_{m}\right)=0
$$

and hence $x_{*}=T x_{*}$. Now, let $m_{J}\left(x_{n}, x_{*}\right)=J\left(x_{*}, x_{n}\right)$; then, by Equation (14), (F1) and Equation (13), we have

$$
J\left(x_{*}, T x_{*}\right)=\lim _{n \rightarrow \infty} J\left(x_{n+1}, T x_{*}\right) \leq \lim _{n \rightarrow \infty} J\left(x_{*}, x_{n}\right)=0
$$


This implies that $x_{*}=T x_{*}$. Therefore, by both cases, we obtain that $x_{*} \in \operatorname{Fix}(T)$.

Finally, we will prove the uniqueness part. Supposing that there exists $z_{*} \in X$ such that $z_{*}=T z_{*}$ and $z_{*} \neq x_{*}$, then $d\left(x_{*}, z_{*}\right)>0$ which implies that $J\left(T x_{*}, T z_{*}\right)>0$. Therefore, by Equation (6),

$$
\begin{aligned}
F\left(J\left(x_{*}, z_{*}\right)\right) & =F\left(J\left(T x_{*}, T z_{*}\right)\right. \\
& \leq F\left(m_{J}\left(x_{*}, z_{*}\right)\right)-\tau .
\end{aligned}
$$

If $m_{J}\left(x_{*}, z_{*}\right)=J\left(x_{*}, z_{*}\right)$, then, by Equation (16), (F1) and $\tau>0$, we have $J\left(x_{*}, z_{*}\right)<J\left(x_{*}, z_{*}\right)$, which is impossible.

If $m_{J}\left(x_{*}, z_{*}\right)=J\left(z_{*}, x_{*}\right)$, then, by definition of $m_{J}$, we get $J\left(z_{*}, x_{*}\right)<J\left(x_{*}, z_{*}\right)$. Hence, from Equation (16) and (F1),

$$
\tau+F\left(J\left(x_{*}, z_{*}\right)\right) \leq F\left(m_{J}\left(x_{*}, z_{*}\right)\right)=F\left(\left(z_{*}, x_{*}\right)\right)<F\left(J\left(x_{*}, z_{*}\right)\right),
$$

which implies that $J\left(x_{*}, z_{*}\right)<J\left(x_{*}, z_{*}\right)$ and it is impossible. Therefore, $z_{*}=x_{*}$ and the proof is complete.

Corollary 1. Let $(X, d)$ be a metric space equipped with a generalized pseudodistance $J$ and let $T: X \rightarrow X$ be a mapping with $\tau \in \mathbb{R}^{+}$. Assume that $X$ is a J-complete and there exists $k \in\left[0, e^{-\tau}\right)$ such that

$$
J(T x, T y) \leq k m_{J} J(x, y)
$$

for all $x, y \in X$. Then, $T$ has a unique fixed point. Furthermore, for any $x_{0} \in X$, define $x_{n}=T x_{n-1}$ for all $n \in \mathbb{N}$, the sequence $\left\{x_{n}\right\}$ converges to $u \in \operatorname{Fix}(T)$ and $\lim _{n \rightarrow \infty} J\left(x_{n}, u\right)=0=\lim _{n \rightarrow \infty} J\left(u, x_{n}\right)$.

Corollary 2. Let $(X, d)$ be a metric space equipped with an w-distance $p$ and let $T: X \rightarrow X$ be a mapping. Assume that $X$ is a w-complete and there exists $F \in \mathcal{F}$ with $\tau \in \mathbb{R}^{+}$such that

$$
p(T x, T y)>0 \Rightarrow \tau+F(p(T x, T y)) \leq F\left(m_{p}(x, y)\right)
$$

for all $x, y \in X$, where $m_{p}(x, y)=\min \{p(x, y), p(y, x)\}$. If $F$ is a continuous from the right, then $T$ has a unique fixed point. Furthermore, for any $x_{0} \in X$, define $x_{n}=T x_{n-1}$ for all $n \in \mathbb{N}$; the sequence $\left\{x_{n}\right\}$ converges to $u \in$ Fix $(T)$ and $\lim _{n \rightarrow \infty} p\left(x_{n}, u\right)=0=\lim _{n \rightarrow \infty} p\left(u, x_{n}\right)$.

Corollary 3. Let $(X, d)$ be a complete metric space and $T: X \rightarrow X$ be a mapping [6]. Assume that there exists a continuous from the right function $F \in \mathcal{F}$ and $\tau \in \mathbb{R}^{+}$such that

$$
\tau+F(d(T x, T y)) \leq F(d(x, y))
$$

for all $x, y \in X$ with $T x \neq T y$. Then, $T$ has a unique fixed point. Furthermore, for any $x_{0} \in X$, define $x_{n}=T x_{n-1}$ for all $n \in \mathbb{N}$, the sequence $\left\{x_{n}\right\}$ converges to $u \in \operatorname{Fix}(T)$.

Next, we provide some illustrative example to support Theorem 1.

Example 1. Let $X=[0,2], E=[0,1]$. Define a metric $d$ on $X$ by $d(x, y)=|x|+|y|$ for all $x, y \in X$. Let J: $X \times X \rightarrow[0, \infty)$ be a generalized pseudodistance on $X$ defined by

$$
J(x, y)= \begin{cases}d(x, y), & E \cap\{x, y\}=\{x, y\} \\ 6, & E \cap\{x, y\} \neq\{x, y\}\end{cases}
$$


Define a mapping $T: X \rightarrow X$ by

$$
T x= \begin{cases}\frac{x}{x+2}, & x \in E ; \\ x-1, & x \notin E .\end{cases}
$$

Now, we will show that the mapping $T$ satisfies (6) with $\tau=0.05>0$ and $F(\alpha)=\ln \alpha$. Then, $F \in \mathcal{F}$ (see [6]), and we distinguish four cases.

Case I Let $x, y \in$ E. Then, the L.H.S. of (6),

$$
\tau+F(J(T x, T y))=0.05+\ln \left(\frac{x}{x+2}+\frac{y}{y+2}\right)
$$

and the R.H.S. of (6),

$$
F\left(m_{J}(x, y)\right)=\ln (x+y) .
$$

Next, we compare L.H.S. and R.H.S. in the 3D view (see Figure 1).

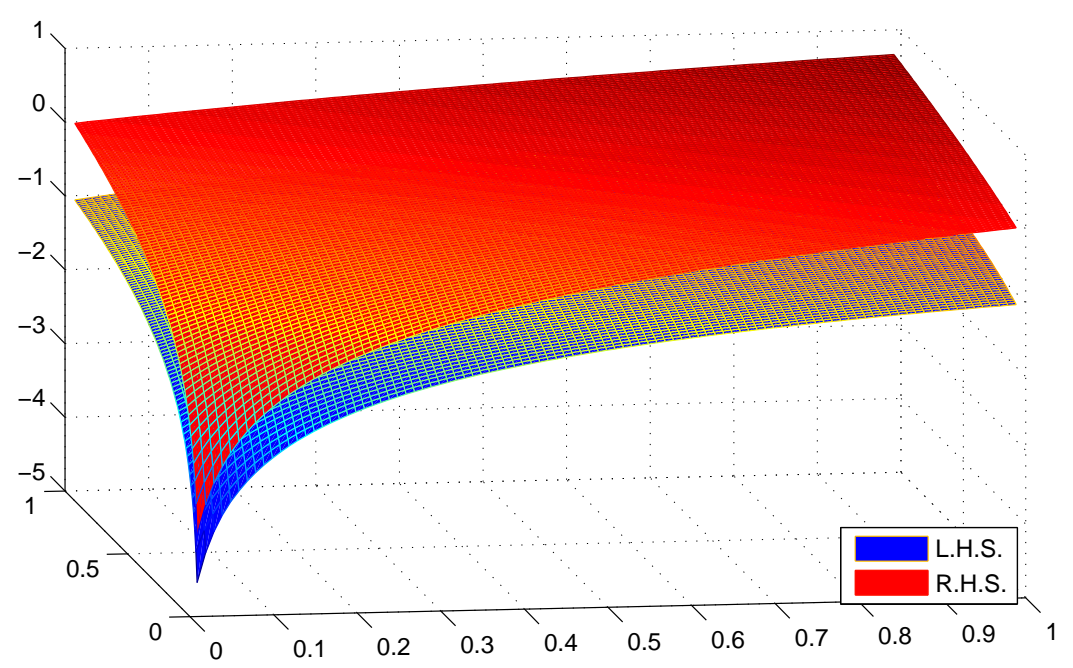

Figure 1. The value of comparison of the L.H.S. and R.H.S. of Equation (6) in the 3D view.

Case II Let $x \in E, y \notin E$. Then,

$$
\begin{aligned}
\tau+F(J(T x, T y)) & =0.05+\ln \left(\frac{x}{x+2}+(y-1)\right) \\
& <0.05+\ln \left(\frac{x}{x+2}+1\right) \\
& <0.05+\ln (2) \\
& =0.7431 \\
& <1.7917 \\
& =\ln (6)=F\left(m_{J}(x, y)\right) .
\end{aligned}
$$

Case III Let $x \notin E, y \in$ E. Similarly to case II, we obtain

$$
\begin{aligned}
\tau+F(J(T x, T y)) & =0.05+\ln \left(\frac{y}{y+2}+(x-1)\right) \\
& \leq \ln (6)=F\left(m_{J}(x, y)\right) .
\end{aligned}
$$

Case IV Let $x \notin E, y \notin E$. Then, 


$$
\begin{aligned}
\tau+F(J(T x, T y)) & =0.05+\ln (x+y-2) \\
& \leq 0.05+\ln (2) \\
& <\ln (6)=F\left(m_{J}(x, y)\right)
\end{aligned}
$$

Therefore, all the hypotheses of Theorem 1 are satisfied. For this, $x=0$ is only a fixed point of $T$ and $J(0,0)=0$.

Theorem 2. Let $(X, d)$ be a metric space equipped with a generalized pseudodistance $J$ and let $T: X \rightarrow X$ be a mapping. Assume that $X$ is a J-complete and there exists $F \in \triangle_{\mathcal{F}}$ and $\tau \in \mathbb{R}^{+}$such that

$$
J(T x, T y)>0 \Rightarrow \tau+F(J(T x, T y)) \leq F(\alpha(J(x, T x)+J(y, T y)))
$$

for all $x, y \in X$, where $\alpha \in\left[0, \frac{1}{2}\right)$. Then, $T$ has a fixed point. Furthermore, for any $x_{0} \in X$, define $x_{n}=T x_{n-1}$ for all $n \in \mathbb{N}$; the sequence $\left\{x_{n}\right\}$ converges to $u \in$ Fix $(T)$ and $\lim _{n \rightarrow \infty} J\left(x_{n}, u\right)=0=\lim _{n \rightarrow \infty} J\left(u, x_{n}\right)$.

Proof. Let $x_{0} \in X$ be arbitrary and define the sequence $\left\{x_{n}\right\}$ by $x_{n}=T x_{n-1}$ for all $n \in \mathbb{N}$. If $x_{n_{0}}=T x_{n_{0}}$, for some $n_{0} \in \mathbb{N}$, then the proof is complete. Suppose that $x_{n} \neq T x_{n}$ for all $n \in \mathbb{N}$. Then, $d\left(x_{n} \cdot x_{n+1}\right)>0$ implies that $J\left(x_{n} \cdot x_{n+1}\right)>0$. Letting $J_{n}:=J\left(x_{n} \cdot x_{n+1}\right)$ for $n \in \mathbb{N} \cup\{0\}$. By Equation (20), we have

$$
\begin{aligned}
\tau+F\left(J_{n}\right) & =\tau+F\left(J\left(T x_{n-1}, T x_{n}\right)\right. \\
& \left.\leq F\left(\alpha\left(J\left(x_{n-1}, T x_{n-1}\right)\right)+J\left(x_{n}, T x_{n}\right)\right)\right) \\
& =F\left(\alpha\left(J_{n-1}+J_{n}\right)\right) .
\end{aligned}
$$

By $(F 1)$, we get

$$
J_{n} \leq \alpha\left(J_{n-1}+J_{n}\right)
$$

and hence

$$
J_{n} \leq \frac{\alpha}{1-\alpha} J_{n-1}
$$

for all $n \in \mathbb{N}$. Therefore,

$$
J_{n} \leq \frac{\alpha}{1-\alpha} J_{n-1} \cdots \leq\left(\frac{\alpha}{1-\alpha}\right)^{n} J_{0} .
$$

Furthermore, for any positive integer $m, n$ such that $m>n$,

$$
\begin{aligned}
J\left(x_{n}, x_{m}\right) & \leq J_{n}+J_{n+1}+\ldots+J_{m-1} \\
& \leq\left(\left(\frac{\alpha}{1-\alpha}\right)^{n}+\left(\frac{\alpha}{1-\alpha}\right)^{n+1}+\ldots+\left(\frac{\alpha}{1-\alpha}\right)^{m-1}\right) J_{0} \\
& \leq \frac{\alpha^{n}}{(1-\alpha)^{n-1}(1-2 \alpha)} J_{0} .
\end{aligned}
$$

Taking $m, n \rightarrow \infty$ reduces to

$$
\lim _{n \rightarrow \infty} \sup _{m>n} J\left(x_{n}, x_{m}\right)=0
$$

Since $J$ is $J$-complete, then the sequence $\left\{x_{n}\right\}$ converges to some element $x_{*} \in X$ with

$$
\lim _{n \rightarrow \infty} J\left(x_{n}, x_{*}\right)=0=\lim _{n \rightarrow \infty} J\left(x_{*}, x_{n}\right) .
$$

Next, we will prove that $x_{*} \in F i x(T)$. If $J\left(T x_{n}, T x_{*}\right)>0$, then by Equation (6),

$$
\begin{aligned}
F\left(J\left(x_{n+1}, T x_{*}\right)\right) & =\tau+F\left(J\left(T x_{n}, T x_{*}\right)\right. \\
& \leq F\left(\alpha\left(J\left(x_{n}, T x_{n}\right)+J\left(x_{*}, T x_{*}\right)\right)\right)-\tau \\
& =F\left(\alpha\left(J\left(x_{n}, x_{n+1}\right)+J\left(x_{*}, T x_{*}\right)\right)\right)-\tau .
\end{aligned}
$$


By $(F 1)$, we get $J\left(x_{n+1}, T x_{*}\right) \leq \alpha\left(J\left(x_{n}, x_{n+1}\right)+J\left(x_{*}, T x_{*}\right)\right)$ and thus

$$
\begin{aligned}
J\left(x_{*}, T x_{*}\right) & =\lim _{n \rightarrow \infty} J\left(x_{n+1}, T x_{*}\right) \\
& \leq \lim _{n \rightarrow \infty} \alpha\left(J\left(x_{n}, x_{n+1}\right)+J\left(x_{*}, T x_{*}\right)\right) \\
& \leq \alpha\left(J\left(x_{*}, x_{*}\right)+J\left(x_{*}, T x_{*}\right)\right) .
\end{aligned}
$$

Consequently,

$$
\begin{aligned}
J\left(x_{*}, T x_{*}\right) & \leq \frac{\alpha}{1-\alpha} J\left(x_{*}, x_{*}\right) \\
& \leq \frac{\alpha}{1-\alpha}\left(J\left(x_{*}, x_{n}\right)+J\left(x_{n}, x_{*}\right)\right) .
\end{aligned}
$$

Then, Equations (24) and (25) imply that $J\left(x_{*}, T x_{*}\right)=0$. Now, for each $n \in \mathbb{N}$, define $x_{m}=x_{n+1}$ and $y_{m}=T x_{*}$ for all $m \in \mathbb{N}$; then,

$$
\lim _{m \rightarrow \infty} J\left(x_{m}, y_{m}\right)=0 .
$$

Equations (23), (26) and (J2) imply that $\lim _{n \rightarrow \infty} d\left(x_{m}, y_{m}\right)=0$. That is,

$$
d\left(x_{*}, T x_{*}\right)=\lim _{n \rightarrow \infty} d\left(x_{n+1}, T x_{*}\right)=\lim _{m \rightarrow \infty} d\left(x_{m}, y_{m}\right)=0 .
$$

Thus, we obtain that $x_{*}=T x_{*}$ and the proof is complete.

Corollary 4. Let $(X, d)$ be a metric space equipped with a generalized pseudodistance $J$ and let $T: X \rightarrow X$ be a mapping. Assume that $X$ is a J-complete and there exists $\lambda \in\left[0, \alpha e^{-\tau}\right)$ such that

$$
J(T x, T y) \leq \lambda(J(x, T x)+J(y, T y))
$$

for all $x, y \in X$. Then, $T$ has a fixed point. Furthermore, for any $x_{0} \in X$ define $x_{n}=T x_{n-1}$ for all $n \in \mathbb{N}$, the sequence $\left\{x_{n}\right\}$ converges to $u \in$ Fix $(T)$ and $\lim _{n \rightarrow \infty} J\left(x_{n}, u\right)=0=\lim _{n \rightarrow \infty} J\left(u, x_{n}\right)$.

Corollary 5. Let $(X, d)$ be a metric space equipped with a w-distance $p$ and let $T: X \rightarrow X$ be a mapping. Assume that $X$ is a w-complete and there exists $F \in \triangle_{\mathcal{F}}$ with $\tau \in \mathbb{R}^{+}$such that

$$
p(T x, T y)>0 \Rightarrow \tau+F(p T x, T y)) \leq F(\alpha(p(x, T x)+p(y, T y)))
$$

for all $x, y \in X$. Then, $T$ has a fixed point. Furthermore, for any $x_{0} \in X$, define $x_{n}=T x_{n-1}$ for all $n \in \mathbb{N}$, the sequence $\left\{x_{n}\right\}$ converges to $u \in$ Fix $(T)$ and $\lim _{n \rightarrow \infty} p\left(x_{n}, u\right)=0=\lim _{n \rightarrow \infty} p\left(u, x_{n}\right)$.

Corollary 6. Let $(X, d)$ be a complete metric space and $T: X \rightarrow X$ be a mapping. Assume that there exists $F \in \triangle_{\mathcal{F}}$ with $\tau \in \mathbb{R}^{+}$such that

$$
\tau+F(d(T x, T y)) \leq F(\alpha(d(x, T x)+d(y, T y)))
$$

for all $x, y \in X$ such that $T x \neq T y$, where $\alpha \in\left[0, \frac{1}{2}\right)$. Then, $T$ has a fixed point. Furthermore, for any $x_{0} \in X$, define $x_{n}=T x_{n-1}$ for all $n \in \mathbb{N}$; the sequence $\left\{x_{n}\right\}$ converges to $u \in$ Fix $(T)$.

Next, we provide some illustrative examples of Theorem 2.

Example 2. Let $X=[0,2]$ and $E=\left\{\frac{1}{n} ; n \in \mathbb{N}\right\} \cup\{0\}$ with the metric defined by $d(x, y)=\max \{x, y\}$ for all $x, y \in X$. Let $J: X \times X \rightarrow[0, \infty)$ be a generalized pseudodistance on $X$ defined by

$$
J(x, y)= \begin{cases}d(x, y), & E \cap\{x, y\}=\{x, y\} \\ 6, & E \cap\{x, y\} \neq\{x, y\}\end{cases}
$$


Let $T: X \rightarrow X$ be defined by

$$
T(x)= \begin{cases}\frac{1}{n^{2}}, & \text { if } x=\frac{1}{n} \\ 0, & \text { otherwise. }\end{cases}
$$

Now, we will show that the mapping $T$ satisfies (20) for $\alpha=0.8, \tau=0.75>0$ and $F(\beta)=\frac{1}{1-e^{\beta}}$. Obviously, $F \in \triangle_{\mathcal{F}}$, we distinguish four cases.

Case I Let $x, y \in E$. If $x=y=0$, then $J(T x, T y)=0$ we will not consider because it does not satisfy the assumption of F. If $x=0$ and $y \in E \backslash\{0\}$ (or $y=0$ and $x \in E \backslash\{0\}$ ), then the L.H.S. of (20),

$$
\tau+F(J(T x, T y))=0.75+F\left(\frac{1}{n^{2}}\right)=0.75+\frac{1}{1-e^{\frac{1}{n^{2}}}},
$$

and the R.H.S. of (20),

$$
F(\alpha(J(x, T x)+J(y, T y)))=\frac{1}{1-e^{0.8 \cdot \frac{1}{n}}} .
$$

Next, we compare R.H.S. and L.H.S. in the 3D view (see Figure 2).

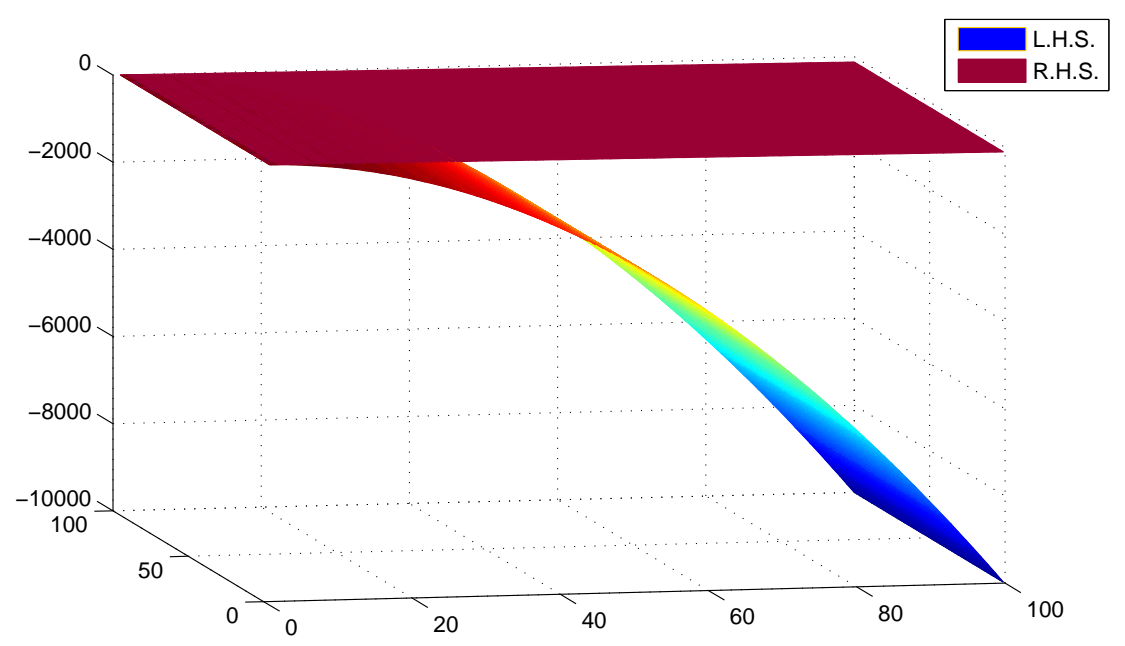

Figure 2. The value of comparison of the L.H.S. and R.H.S. of Equation (20) in the 3D view.

Next, let $x=\frac{1}{n}$ and $y=\frac{1}{m}$ for some $m, n \in \mathbb{N}$ such that $m>n$. Then, for the L.H.S. of Equation (20),

$$
\tau+F(J(T x, T y))=0.75+F\left(\frac{1}{n^{2}}\right)=0.75+\frac{1}{1-e^{\frac{1}{n^{2}}}},
$$

and for the R.H.S. of (20),

$$
F(\alpha(J(x, T x)+J(y, T y)))=\frac{1}{1-e^{0.8 \cdot\left(\frac{1}{n}+\frac{1}{m}\right)}} .
$$

In the following figures (Figure 3), we compare the L.H.S. and R.H.S. in the 3D view.

Case II Let $x \in E$, and let $y \notin E$. If $x=0$, then $T x=T y=0$ and $J(T x, T y)=0$ we will not consider because it does not satisfy the assumption of $F$, so we are done. Suppose that $x \in E \backslash\{0\}$, then

$$
0.75+F\left(\frac{1}{n^{2}}\right)=0.75+\frac{1}{1-e^{\frac{1}{n^{2}}}}
$$

and for the R.H.S. of (20), 


$$
F(\alpha(J(x, T x)+J(y, T y)))=\frac{1}{1-e^{0.8 \cdot\left(\frac{1}{n}+6\right)}}
$$

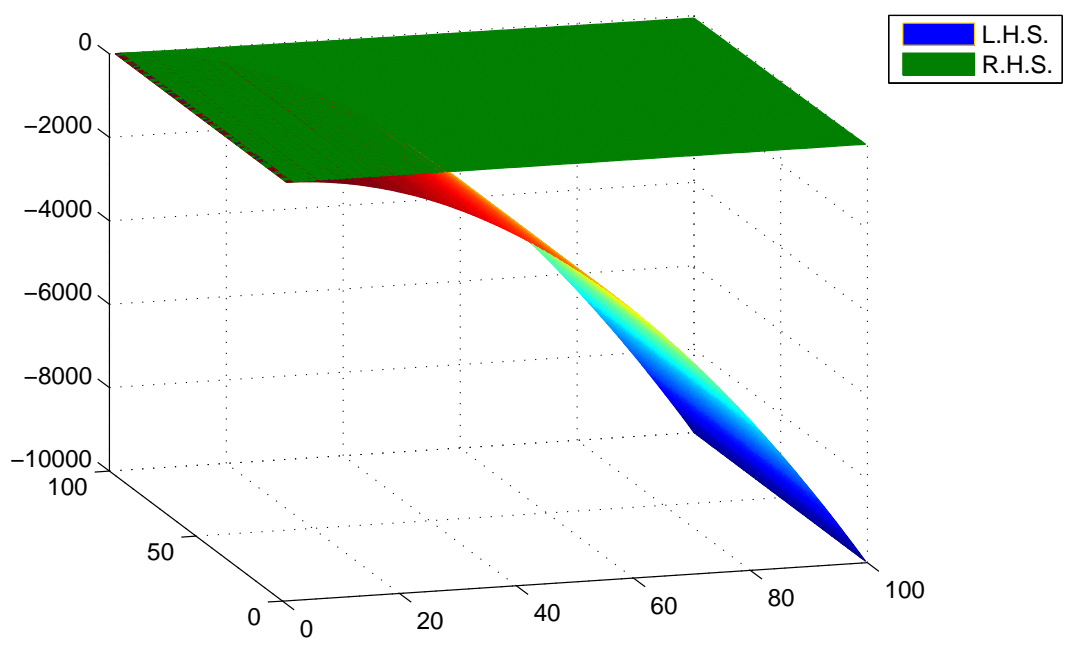

Figure 3. The value of the comparison of L.H.S. and R.H.S. of Equation (20) in the 3D view.

In the following figures (Figure 4), we compare R.H.S. and L.H.S. in the 3D view.

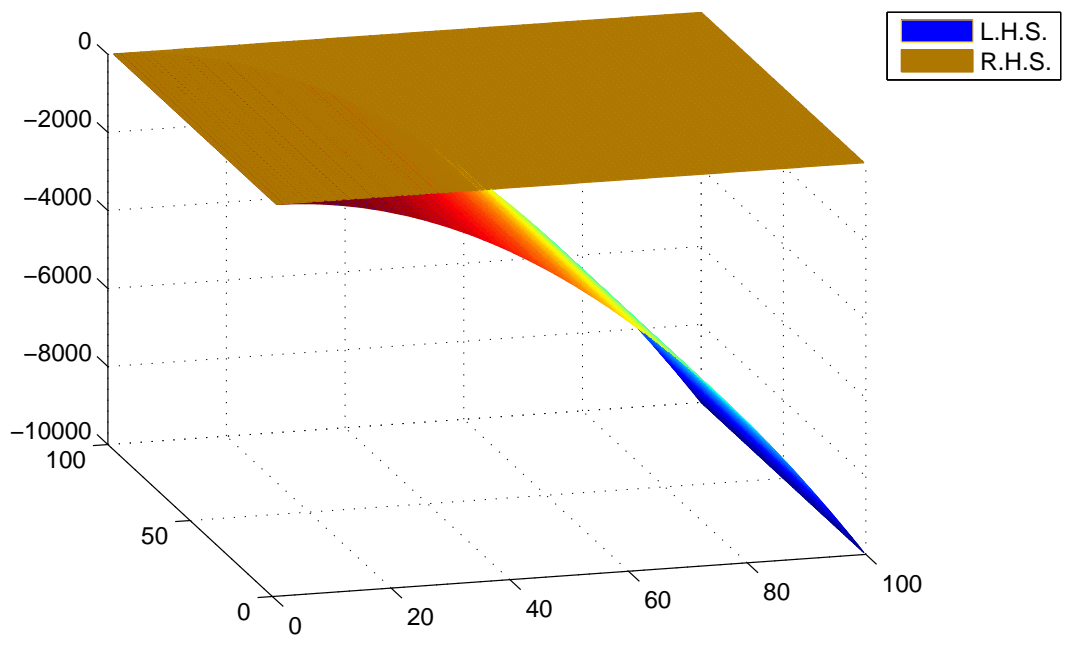

Figure 4. The value of comparison of L.H.S. and R.H.S. of Equation (20) in the 3D view.

Case III Let $x \notin E$, and let $y \in E$. This is similar to case II.

Case IV Let $x, y \notin E$. Then, $J(T x, T y)=0$. Thus, it does not satisfy the condition of $F$.

Thus, all the conditions of Theorem 2 are satisfied. For this, $x=0$ and $x=1$ are fixed points of T. Moreover, $J(0,0)=0$ and $J(1,1)=0$.

\section{Applications}

In this section, we apply our result to prove the existence theorem for the solution of the second order differential equation of the form:

$$
\left\{\begin{array}{l}
\frac{d^{2} u}{d t^{2}}+\frac{c}{m} \frac{d u}{d t}=K(t, u(t)), \\
u(0)=0, u^{\prime}(0)=a,
\end{array}\right.
$$


where $I>0$ and $K$ is a continuous function from $[0, I] \times \mathbb{R}^{+}$to $\mathbb{R}$.

The above differential equation shows the engineering problem of activation of a spring that is affected by an exterior force. It is well known that the Equation (30) is equivalent to the integral equation as follows:

$$
u(t)=\int_{0}^{t} G(t, s) K(s, u(s)) d s, \quad t \in[0, I],
$$

when $G(t, s)$ is the green function defined by

$$
G(t, s)= \begin{cases}(t-s) e^{\tau(t-s)}, & \text { if } 0 \leq s \leq t \leq I, \\ 0, & \text { if } 0 \leq t \leq s \leq I,\end{cases}
$$

with $\tau>0$ is a constant, in terms of $c$ and $m$ in Equation (30).

Let $X:=C\left([0, I], \mathbb{R}^{+}\right)$and $\tau>0$, when $C\left([0, I], \mathbb{R}^{+}\right)$is the set of all continuous functions from $[0, I]$ in to $\mathbb{R}^{+}$. For $u \in X$, we define

$$
\|u\|_{\tau}=\sup _{t \in[0, I]}\left\{|u(t)| e^{-2 \tau t}\right\}
$$

and the $w$-distance $p: X \times X \rightarrow[0, \infty)$ is defined by

$$
p(x, y)=\min \left\{\|x\|_{\tau},\|y\|_{\tau}\right\}
$$

for all $x, y \in X$. Consider a function $T: X \rightarrow X$ defined as follows:

$$
T(u(t))=\int_{0}^{t} G(t, s) K(s, u(s)) d s \text { where } t \in[0, I]
$$

for all $x \in X$.

Clearly, the existence of a fixed point under a mapping $T$ is equivalent to the existence of a solution of Equation (31).

Theorem 3. Consider the nonlinear integral Equation (31). Assume that the following three conditions hold:

(A) $K$ is continuous and increasing function;

(B) there exists $\tau>0$ such that

$$
|K(s, u)| \leq \tau^{2} e^{-\tau} u,
$$

where $s \in[0,1]$ and $u \in \mathbb{R}^{+}$;

(C) $T: X \rightarrow X$ satisfies (33).

Then, the Equation (31) has a solution.

Proof. Now, we show that the function $T$ defined as Equation (33) satisfies Equation (18). For this, we consider

$$
\begin{aligned}
|T(u(s))| & =\int_{0}^{t} G(t, s)|K(s, u(s))| d s \\
& \leq \int_{0}^{t} G(t, s) \tau^{2} e^{-\tau}|u(s)| d s \quad \text { (by using conditions (A) \& (B)) } \\
& =\int_{0}^{t} \tau^{2} e^{-\tau}(t-s) e^{\tau(t-s)} e^{2 \tau s}\|u\|_{\tau} d s \\
& =\tau^{2} e^{-\tau+\tau t}\|u\|_{\tau} \int_{0}^{t}(t-s) e^{\tau s} d s \\
& =e^{-\tau}\|u\|_{\tau} e^{2 \tau t}\left[1-\tau t e^{-\tau t}-e^{-\tau t}\right]
\end{aligned}
$$


Because $\left[1-\tau t e^{-\tau t}-e^{-\tau t}\right] \leq 1$, we have

$$
\| T\left(u(s)\left\|_{\tau} \leq e^{-\tau}\right\| u \|_{\tau} .\right.
$$

Similarly,

$$
\|T(v(s))\|_{\tau} \leq e^{-\tau}\|v\|_{\tau}
$$

Therefore,

$$
\begin{aligned}
p(T u, T v) & =\min \left\{\|T u\|_{\tau},\|T v\|_{\tau}\right\} \\
& \leq e^{-\tau} \min \left\{\|u\|_{\tau},\|v\|_{\tau}\right\} \\
& =e^{-\tau} \min \left\{\min \left\{\|u\|_{\tau},\|v\|_{\tau}\right\}, \min \left\{\|v\|_{\tau},\|u\|_{\tau}\right\}\right\} \\
& =e^{-\tau} m_{p}(u, v)
\end{aligned}
$$

for all $u, v \in X$.

By taking the logarithm, from the above inequality, we can write

$$
\tau+\ln (p(T u, T v)) \leq \ln \left(m_{p}(u, v)\right) .
$$

By considering the function $F$ defined in Remark 3, we obtain that

$$
\tau+F(p(T x, T y)) \leq F\left(m_{p}(x, y)\right)
$$

and hence $T$ satisfies (18). Therefore, all the hypotheses of Corollary 2 are satisfied, and hence by Corollary 2, Equation (31) has a solution. This means that the second order differential Equation (30) has a solution.

Author Contributions: Writing of the original draft: S.S. and W.S., Writing, review and editing: C.M.

Funding: This research was funded by the Kasetsart University Research and Development Institute (KURDI). The third author was supported by the Thailand Research Fund and the Office of the Higher Education Commission (Grant No. MRG6080244).

Acknowledgments: The authors thank Editors and Referee(s) for their valuable comments and suggestions, which were very useful to improve the paper significantly. The first author would like to thank the Kasetsart University Research and Development Institute (KURDI) for financial support.

Conflicts of Interest: The authors declare no conflict of interest.

\section{References}

1. Kada, O.; Suzuki, T.; Takahashi, W. Nonconvex minimization theorems and fixed point theorems in complete metric spaces. Math. Jpn. 1996, 44, 381-391.

2. Włodarczyk, K.; Plebaniak, R. Maximality principle and general results of Ekeland and Caristi types without lower semicontinuity assumptions in cone uniform spaces with generalized pseudodistances. Fixed Point Theory Appl. 2010, 2010, 175453. [CrossRef]

3. Włodarczyk, K.; Plebaniak, R. A fixed point theorem of Subrahmanyam type in uniform spaces with generalized pseudodistances. Appl. Math. Lett. 2011, 24, 325-328. [CrossRef]

4. Włodarczyk, K.; Plebaniak, R. Contractions of Banach, Tarafdar, Meir - Keeler, Ćirić-Jachymski-Matkowski and Suzuki types and fixed points in uniform spaces with generalized pseudodistances. J. Math. Anal. Appl. 2013, 404, 338-350. [CrossRef]

5. Mongkolkeha, C.; Kumam, P. Some Existence of coincidence point and approximate solution method for generalized weak contraction in b-generalized pseudodistance functions. Filomat 2017, 31, 6185-6203. [CrossRef] 
6. Wardowski, D. Fixed points of new type of contractive mappings in complete metric space. Fixed Point Theory Appl. 2012, 2012, 94. [CrossRef]

7. Piri, H.; Kumam, P. Some fixed point theorems concerning F-contraction in complete metric spaces. Fixed Point Theory Appl. 2014, 2014, 210. [CrossRef]

8. Budhia, L.; Kumam, P.; Martinez-Moreno, J.; Gopal, D. Extensions of almost- $F$ and $F$-Suzuki contractions with graph and some applications to fractional calculus. Fixed Point Theory Appl. 2016, 2016, 2. [CrossRef]

9. Lukács, A.; Kájantó, K. Fixed point theorems for various type of $F$-contractions in complete metric spaces. Fixed Point Theory 2018, 19, 321-334. [CrossRef]

10. Secelean, N.A. Iterated function systems consisting of F-contractions. Fixed Point Theory Appl. 2013, 2013, 277. [CrossRef]

11. Singh, D.; Joshi, V.; Imdad, M.; Kumam, P. Fixed point theorems via generalized F-contractions with applications to functional equations occurring in dynamic programming. J. Fixed Point Theory Appl. 2017, 19, 1453-1479. [CrossRef]

12. Takahashi, W. Nonlinear Functional Analysis Fixed Point Theory and its Applications; Yokahama Publishers: Yokahama, Japan, 2000.

13. Plebaniak, R. On best proximity points for set-valued contractions of Nadler type with respect to $b$-generalized pseudodistances in $b$-metric spaces. Fixed Point Theory Appl. 2014, 2014, 39. [CrossRef]

14. Plebaniak, R. New generalized pseudodistance and coincidence point theorem in a $b$-metric spaces. Fixed Point Theory Appl. 2013, 2013, 270. [CrossRef]

(C) 2018 by the authors. Licensee MDPI, Basel, Switzerland. This article is an open access article distributed under the terms and conditions of the Creative Commons Attribution (CC BY) license (http:// creativecommons.org/licenses/by/4.0/). 